\title{
Alterations of left ventricular function in women with insulin-dependent diabetes mellitus during pregnancy
}

\author{
C. M. Schannwell ${ }^{1}$, M. Schneppenheim ${ }^{1}$, S. M. Perings ${ }^{1}$, T. Zimmermann ${ }^{2}$, G. Plehn ${ }^{1}$, B. E. Strauer ${ }^{1}$ \\ ${ }^{1}$ Clinic of Cardiology, Pneumology and Angiology, Heinrich-Heine University, Düsseldorf, Germany \\ ${ }^{2}$ Clinic of Gynaecology and Obstetrics, Heinrich-Heine University, Düsseldorf, Germany
}

\begin{abstract}
Aims/hypothesis. During pregnancy, eminent cardiovascular changes occur. The aim of the following study was to investigate the course of haemodynamic parameters under the increased volume load during pregnancy and delivery in women with insulin-dependent diabetes mellitus.

Methods. We examined 51 pregnant diabetic women and 51 healthy pregnant women. The control group consisted of 51 healthy non-pregnant women. In all women, left ventricular mass and fractional shortening were calculated. To evaluate left ventricular diastolic function, mitral inflow and pulmonary venous flow profiles were analysed.

Results. During pregnancy left ventricular mass increased, fractional shortening decreased and diastolic dysfunction was found. While the healthy pregnant women developed signs of disturbed relaxation during pregnancy, pregnant diabetic women showed signs of a disturbed relaxation at the beginning of gestation. Of
\end{abstract}

the pregnant diabetic women, 29 developed a restrictive filling pattern at the 24th week of gestation. The remaining 22 diabetic women had a comparable restrictive filling pattern only during vaginal delivery. In 10 of the 29 pregnant diabetic women dangerous complications were documented, while there were no complications in the healthy pregnant women and the other 22 diabetic pregnant women.

Conclusion/interpretation. In healthy women pregnancy results in a reversible physiologic left ventricular hypertrophy, a disturbed relaxation pattern and a temporary decrease of left ventricular systolic function. In contrast, pregnant diabetic women showed a delayed relaxation at the beginning of pregnancy and developed a restrictive filling pattern. The early development of a restrictive filling pattern could indicate complications during delivery in pregnant diabetic women. [Diabetologia (2003) 46:267-275]

Keywords Diastolic function, pregnancy, Type 1 diabetes mellitus, echocardiography.
Pregnancy causes haemodynamic changes in the cardiovascular system due to the physiological volumeoverload [1]. In former studies, changes in haemody-

Received: 27 May 2002 / Revised: 27 August 2002

Published online: 14 February 2003

C Springer-Verlag 2003

Corresponding author: Dr. C. M. Schannwell, Clinic of Cardiology, Pneumology and Angiology, Heinrich-Heine University, Moorenstraße 5, 40225 Düsseldorf, Germany

E-mail: schannw@uni-duesseldorf.de

Abbreviations: at, acceleration time; dt, deceleration time; $\mathrm{VE}$, peak mitral flow velocity in early diastole; VA, peak mitral flow velocity at atrial contraction. namic parameters in the maternal cardiovascular system were evaluated noninvasively by echocardiography $[2,3]$. In earlier studies, echocardiography was frequently applied in order to describe left ventricular systolic function and cardiac haemodynamics during pregnancy $[1,4,5,6,7,8]$, since it allows assessment of cardiac haemodynamics without endangering the pregnancy. However little data on left ventricular diastolic function during pregnancy has been assessed. Our own working group was able to show that an uncomplicated pregnancy in healthy women with normal left ventricular function causes reversible haemodynamic changes with a passager restrictive filling pattern at delivery [8]. In patients with insulin-dependent 
diabetes mellitus, assessment of left ventricular diastolic functional parameters leads to an early recognition of diastolic dysfunction while systolic ventricular function was normal [9]. The aim of this prospective investigation was to evaluate whether the natural volume-overload of pregnancy causes changes in left ventricular systolic and diastolic function in young women with Type 1 diabetes mellitus during pregnancy in comparison to pregnant healthy women.

\section{Subjects and Methods}

Study participants. Between June 1998 and August 2000, 51 pregnant women with Type 1 diabetes mellitus were included in the study. The mean age of the women was $24 \pm 6$ years with a range from 18 to 31 years. Inclusion criteria were an insulintherapy enduring for at least 48 months and a clinically inconspicuous cardiopulmonary result. Late diabetic complications and pulmonary disease were exclusion criteria. All included patients with diabetes mellitus were non-smokers and there was no albuminuria in all examined women. The retention values were within normal range (serum creatinine, serum urea, endogenous creatinine clearance). The velocity of nerve conduction measured at the nervus peronaeus showed normal results.

The control group consisted of 51 healthy pregnant women of corresponding age (mean age $26 \pm 4$ years; range from 18 to 32 years) as well as 51 healthy non-pregnant women of corresponding age (age: $25 \pm 4$ years, range from 19 to 30 years). Using noninvasive diagnostic criteria (electrocardiography, ergometry, spirometry and laboratory analysis), cardiac pulmonary diseases and nephrological diseases as well as high-risk pregnancies (primigravida under 16 and over 34 years of age) and multigravida were excluded from the study. All pregnant women and the attending gynaecologists consented to take part in the study.

Study design. After the diagnosis of pregnancy, additional examinations were carried out: a general clinical examination, weight and blood pressure measurements, urine analysis, blood-typing, and blood analysis for rubella and syphilis. Furthermore, a 12-lead electrocardiography and a transthoracic echocardiography were carried out. In the 9th, 24th, and 33rd week of pregnancy as well as 8 weeks after delivery the clinical and echocardiographic follow-up examinations were carried out.

Clinical examination. To be able to evaluate the course of pregnancy weight, blood pressure, urine analysis and subjective discomfort of the pregnant women were obligatorily recorded. The gestational age was established by clinical and sonographic criteria by the attending gynaecologist.

Laboratory analyses. As criterion for the effective blood glucose regulation, the glycolysed haemoglobin $\left(\mathrm{HbA}_{1 \mathrm{c}}\right)$ was measured. In this examination, the arithmetic mean of the $\mathrm{HbA}_{1 \mathrm{c}}$ values of the previous 2 years served as a measure for the regulation quality. Regulation of the $\mathrm{HbA}_{1 c}$-value ensued column-chromatographically (Bio-wheel column-test; Biowheel Laboratories; Standard values 5.00-8.00\%).

Echocardiographic examination. Ultrasonic examinations were carried out by means of a Toshiba ultrasonic device, model
SSH-160 (Toshiba Corporation Otawara, Japan). A complete two-dimensional, M-mode and Doppler echocardiographic examination was carried out in all women. M-mode measurements of the left atrium and left ventricle were made according to the guidelines of the American Society of Echocardiography [10]. The dimensions of the left atrium, the left chamber, and the systolic and diastolic wall thicknesses were routinely measured. Left ventricular mass and left ventricular ejection fraction were calculated $[11,12]$. Left ventricular stroke volume, cardiac output, cardiac index, and systemic vascular resistance were calculated automatically.

Left ventricular inflow velocities were measured by the pulsed wave Doppler technique in the apical four-chamber view under two-dimensional guidance [13]. The sampling volume was placed near the tips of the mitral leaflets in order to measure the mitral inflow velocities [2]. The following parameters of diastolic function were evaluated: peak mitral flow velocity in early diastole $(\mathrm{m} / \mathrm{s})$; peak mitral flow velocity at atrial contraction $(\mathrm{m} / \mathrm{s})$; ratio of peak mitral flow velocity in early diastole and peak mitral flow velocity at atrial contraction; acceleration time (ms); deceleration time ( $\mathrm{ms}$ ) and isovolumetric relaxation time (ms). Three abnormal left ventricular filling patterns are recognized. The least abnormal and most common is termed "impaired relaxation", resulting from reduced filling in early diastole, a reduced mitral E-to A-wave ratio, increased A-wave amplitude and filling caused by atrial contraction. With disease progression, left ventricular compliance becomes reduced and left atrial pressure increases, which counteracts the impaired left ventricular relaxation. The increased early transmitral pressure gradient results in an left ventricular filling pattern that seems normal but is actually pseudonormal. This term indicates that despite the normal mitral E- to A-wave ratio, abnormalities of left ventricular relaxation and left ventricular compliance are present. Finally, in patients with advanced disease and a severe decrease in left ventricular compliance, high pressures cause left ventricular filling to become restrictive, with blood rapidly entering a slowly relaxing ventricle in early diastole only to be abruptly decelerated. With an increase in early left ventricular diastolic pressure the left atrium is dilated and hypocontractile with little additional filling at atrial contraction.

Using colour Doppler the flow of the right superior pulmonary vein was found. A $5 \mathrm{~mm}$ sample volume was than positioned at the level of the orifice of the right superior pulmonary vein in the left atrium and the velocity of the pulmonary vein flow was documented [14]. The following parameters were assessed: pulmonary venous flow velocity in systole $(\mathrm{m} / \mathrm{s})$; pulmonary venous flow velocity in diastole $(\mathrm{m} / \mathrm{s})$; reverse pulmonary venous flow velocity at atrial contraction $(\mathrm{m} / \mathrm{s})$ and A-wave duration (ms).

Recordings were done at the end of normal expiration in order to eliminate the effects of respiration on the parameters studied [15]. The respective mean value from five consecutive heart cycles was assessed in order to determine various functional parameters.

Statistics. The statistical assessment has been carried out by means of a software program (SPSS, Version 6.1.). The data were provided as mean values with standard deviation. The single groups were compared among each other by means of the Mann-Whitney U test, while Spearman's test was applied for correlations and ANOVA was carried out to compare the results. A significant group divergence was assumed at an error likelihood of less than $5 \%$ with regard to the equality of the examined groups. A $p$ value of less than 0.05 was considered statistically significant. 


\section{Results}

Demographic data. Table 1 gives the most important baseline characteristics. All three study groups showed comparable values regarding body surface area and age. Pregnant diabetic women and pregnant healthy women had comparable duration of pregnancy $(40 \pm 7$ versus $40 \pm 5$ weeks; $p=$ n.s. $)$. Of the pregnant diabetic women, $28(55 \%)$ and 26 healthy pregnant women without diabetes mellitus (51\%) were primigravida $(p=\mathrm{n} . \mathrm{s}$.) (Table 1). There were no statistically significant differences among the three groups concerning concentrations of fasting blood sugar, glycosylated haemoglobin, total cholesterol, HDL and LDL cholesterol as well as triglycerides.

There was a comparable increase of body weight during the course of pregnancy in both groups. Eight weeks after delivery, the initial weight was reached in both groups and was comparable with the non-pregnant women (Table 2).

Haemodynamic parameters. The systolic and diastolic blood pressure values were comparable during the course of pregnancy in both groups.

Cardiac output was increased until delivery. During pregnancy the cardiac index increased from the first trimester to delivery by $26 \%$ from $2.6 \pm 0.5$ to $3.5 \pm 0.8$ $1 \times \mathrm{min}^{-1} \times \mathrm{m}^{-2}(p<0.01)$ in pregnant healthy women, and by $35 \%$ from $2.4 \pm 0.5$ to $3.5 \pm 0.41 \times \mathrm{min}^{-1} \times \mathrm{m}^{-2}(p<0.01)$ in pregnant women with Type 1 diabetes. This increase can be attributed to an increase of the heart rate as well as to a non-significant increase of the stroke volume in both groups (Table 2). The systemic vascular resistance fell in the third trimester and during delivery to the lowest value, while the values were within normal scope at any time. In comparison to the healthy pregnant women, the values for the systemic vascular resistance showed a change in the direction of higher values in the pregnant diabetic women in each trimester without statistical relevance.
Haemoglobin and haematocrit reached their lowest values in all pregnant women in the third trimester and during delivery and reached (yet incompletely) initial values 8 weeks after delivery (Table 2).

Echocardiography. The echocardiographically measured enddiastolic and endsystolic left ventricular dimensions did not change during pregnancy, whether or not the pregnant woman had Type 1 diabetes. During pregnancy in both groups an increase in atrial diameter could be documented.

In all three groups, fractional shortening and left ventricular ejection fraction were within normal range as an expression of normal left ventricular systolic function. The fractional shortening and ejection fraction (EF) decreased in both groups of pregnant women shortly before delivery (EF: pregnant diabetic women from $63 \pm 4$ to $56 \pm 4 \%, p<0.01$ and pregnant women without diabetes from $64 \pm 5$ to $55 \pm 5 \% ; p<0.01)$ in order to reach the initial values postpartum. Between the two groups, there were no relevant differences (Table 3).

Furthermore, an increase in left ventricular mass as well as left ventricular mass index could be documented during pregnancy in both groups. Even 8 weeks after delivery, there was a normalization of the left ventricular wall thickness as well as a reduction of the left ventricular mass index in both groups (Table 3).

Left ventricular diastolic functional parameters showed a divergent behaviour between pregnant women with and without diabetes. Healthy pregnant women developed a disturbed relaxation filling pattern (Table 4) with an increase in the maximum early diastolic inflow velocity $\left(\mathrm{V}_{\mathrm{E}}\right)$ from $0.89 \pm 0.06$ to $0.91 \pm 0.05 \mathrm{~m} / \mathrm{s}(p=$ n.s. $)$ in the second trimester and the maximum late diastolic inflow velocity $\left(\mathrm{V}_{\mathrm{A}}\right)$ at delivery from $0.51 \pm 0.06$ to $0.70 \pm 0.06 \mathrm{~m} / \mathrm{s}(p<0.01)$. The $\mathrm{V}_{\mathrm{E}} / \mathrm{V}_{\mathrm{A}}$ ratio decreased during pregnancy because of the increase of the A-wave. No changes were registered during pregnancy regarding acceleration time. Furthermore, there was a prolongation in isovolumet-
Table 1. Patient characteristics

BSA = body surface area n.s. = not significant

\begin{tabular}{lcccc}
\hline & $\begin{array}{l}\text { Healthy } \\
\text { non-pregnant } \\
\text { women }\end{array}$ & $\begin{array}{l}\text { Healthy } \\
\text { pregnant } \\
\text { women }\end{array}$ & $\begin{array}{c}\text { Pregnant } \\
\text { diabetic } \\
\text { women }\end{array}$ & $p$ \\
\hline Number [n] & 51 & 51 & 51 & n.s. \\
Age [years] & $25 \pm 4$ & $26 \pm 4$ & $24 \pm 6$ & n.s. \\
BSA [m²] & $1.69 \pm 0.12$ & $1.68 \pm 0.14$ & $1.69 \pm 0.16$ & n.s. \\
Primigravida [\%] & & 51 & 55 & n.s. \\
Primigravida [n] & & 26 & 28 & n.s. \\
Duration of pregnancy [months] & $92 \pm 11$ & $80 \pm 5$ & $40 \pm 4$ & n.s. \\
Fasting blood sugar & $5.8 \pm 0.8$ & $6.1 \pm 0.6$ & $68 \pm 9$ & n.s. \\
Glycosylated haemoglobin & $211 \pm 15$ & $198 \pm 16$ & $217 \pm 10$ & n.s. \\
Cholesterol [mg/dl] & $64 \pm 11$ & $61 \pm 8$ & $59 \pm 12$ & n.s. \\
HDL-cholesterol [mg/dl] & $142 \pm 18$ & $136 \pm 12$ & $151 \pm 20$ & n.s. \\
LDL-cholesterol [mg/dl] & $248 \pm 24$ & $213 \pm 17$ & $206 \pm 19$ & n.s. \\
Triglycerides [mg/dl] & & &
\end{tabular}



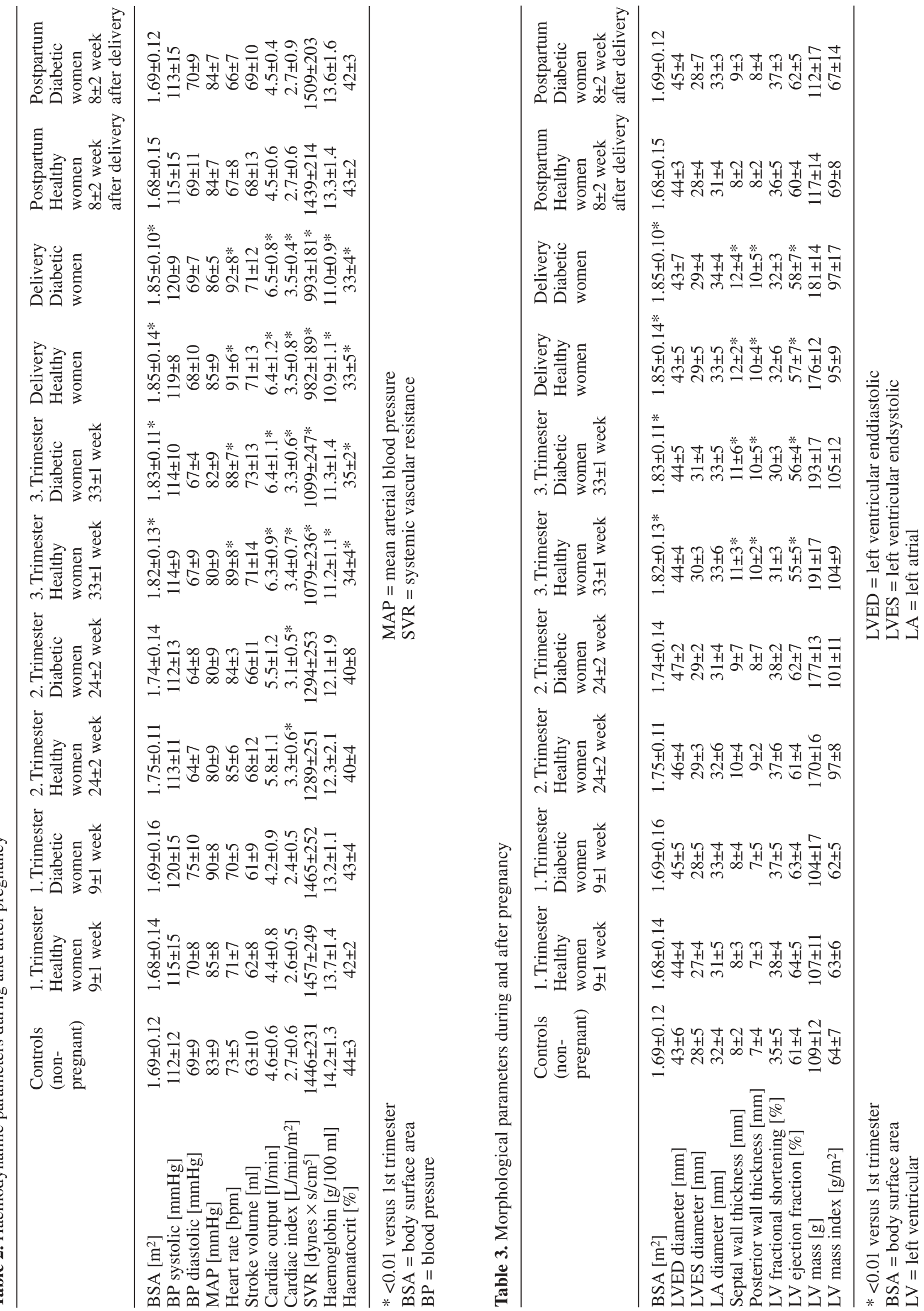


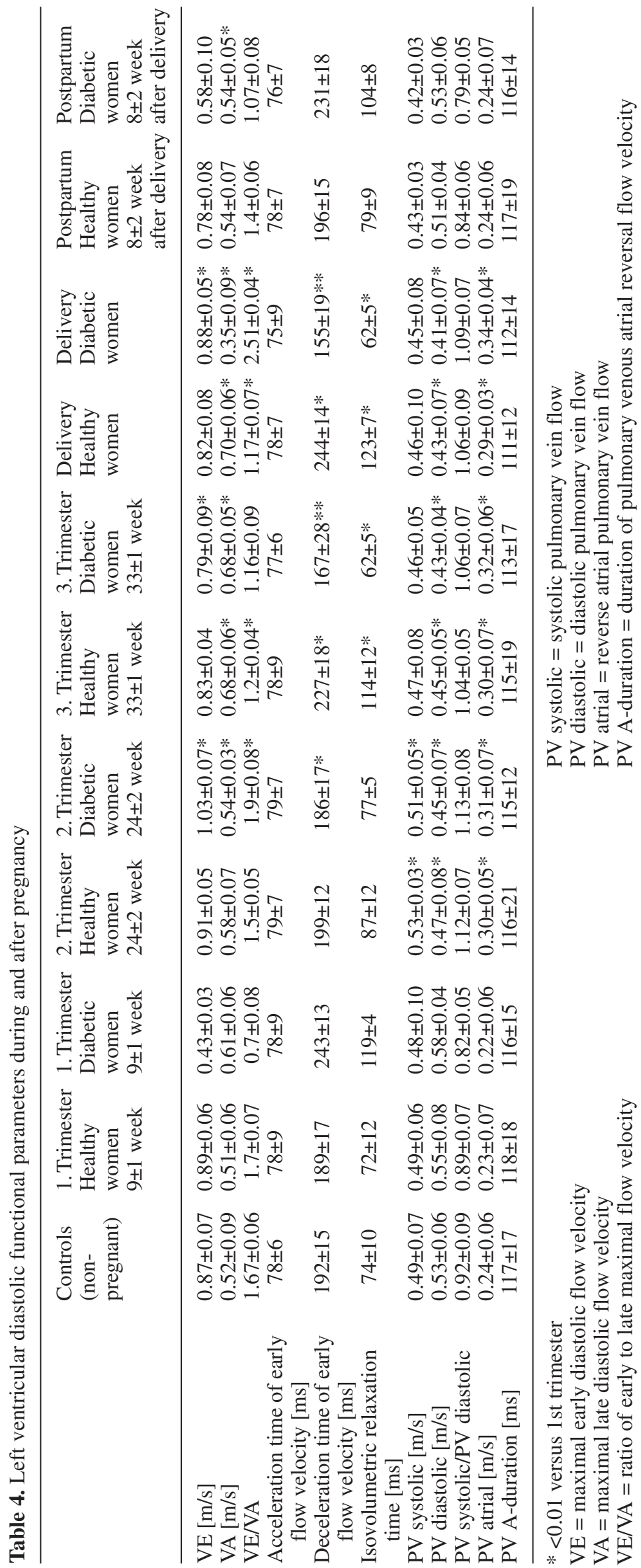




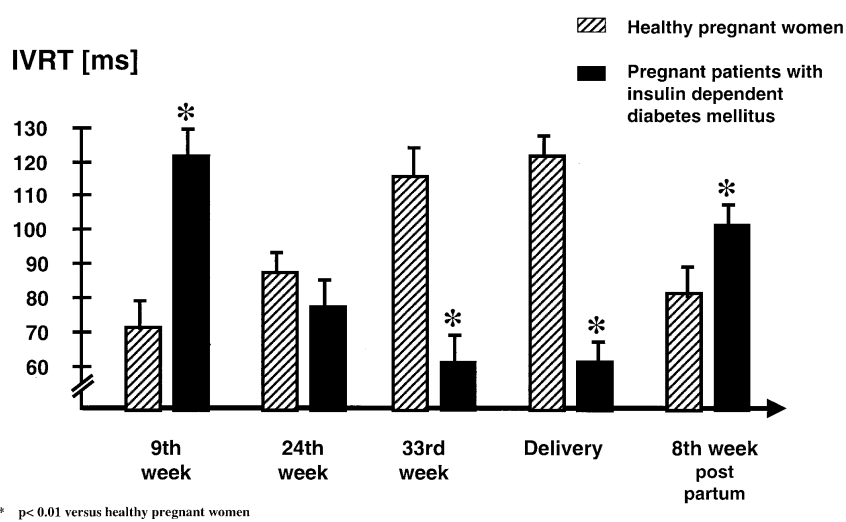

Fig. 1. Isovolumetric relaxation time in the course of pregnancy
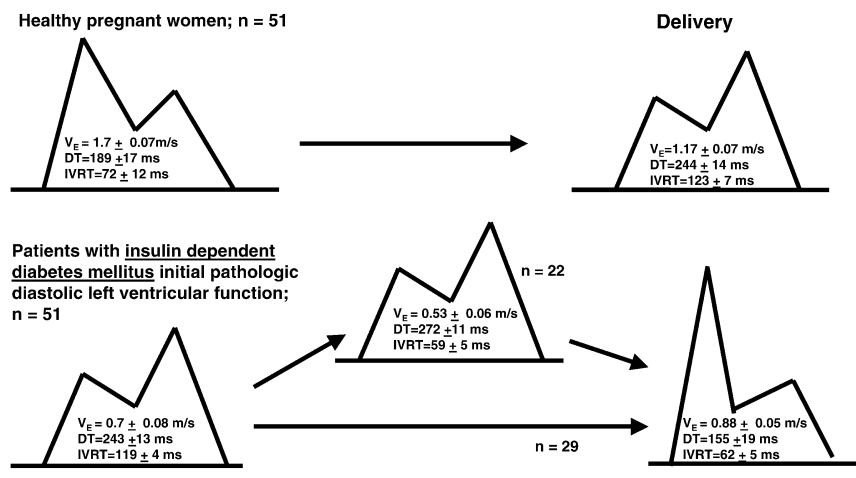

Fig. 2. Alterations of the left ventricular diastolic filling pattern during pregnancy (in healthy pregnant women developed a disturbed relaxation filling pattern, pregnant diabetic women already had a disturbed relaxation filling pattern at the initial examination, in 29 pregnant diabetic patients a restrictive diastolic filling developed from the 24th week of gestation, while the remaining 22 pregnant women developed a restrictive filling pattern only under labour and delivery. $\mathrm{V}_{\mathrm{E}}$, peak mitral flow velocity in early diastole; dt, deceleration time; IVRT, isovolumetric relaxation time)

ric relaxation time (Fig. 1) and deceleration time at delivery compared with the initial values (Table 4).

In contrast, a disturbed relaxation filling pattern was documented in the pregnant diabetic women already at the initial examination (9th week of gestation) with a decreased early diastolic inflow velocity, increased late diastolic inflow velocity and a prolonged deceleration and isovolumetric relaxation time (Table 4). In 29 pregnant diabetic patients a restrictive diastolic filling pattern developed from the 24th week of gestation, while the remaining 22 pregnant diabetic women developed a restrictive filling pattern only under labour and delivery (Fig. 2). A restrictive filling pattern is defined as an elevated early diastolic inflow velocity, decreased late diastolic inflow velocity and reduced deceleration and isovolumetric relaxation time (2nd trimester: 29 diabetic patients with restrictive filling pattern: $\mathrm{V}_{\mathrm{E}} 1.5 \pm 0.07 \mathrm{~m} / \mathrm{s} ; \mathrm{V}_{\mathrm{A}} 0.45 \pm 0.06$ $\mathrm{m} / \mathrm{s}$; deceleration time $143 \pm 11 \mathrm{~ms}$; isovolumetric relaxation time $59 \pm 7 \mathrm{~ms}$ versus 22 diabetic patients with disturbed relaxation pattern $\mathrm{V}_{\mathrm{E}} 0.42 \pm 0.04 \mathrm{~m} / \mathrm{s} ; \mathrm{V}_{\mathrm{A}}$ $0.67 \pm 0.05 \mathrm{~m} / \mathrm{s}$; deceleration time $244 \pm 16 \mathrm{~ms}$; isovolumetric relaxation time $107 \pm 9 \mathrm{~ms}$ ).

During pregnancy there was a comparable non-significant increase in the systolic pulmonary venous flow with a maximum in the 2nd trimester in both groups (Table 4). The diastolic pulmonary venous flow clearly decreased in the 2nd and 3rd trimester. The atrial pulmonary venous flow increased in the course of the pregnancy by about $23 \%$ without any change regarding duration in the atrial pulmonary venous flow. As a measure for mean left atrial pressure, the ratio of systolic and diastolic pulmonary venous flow was calculated in all patients. However, there were no relevant differences between the two patient groups, regarding the pulmonary venous flows. Furthermore, there was no statistically relevant difference concerning the initial data in the age-corresponding normal control subjects, either.

Clinical course of pregnancy. Pregnant women with Type 1 diabetes and a disturbed relaxation pattern during pregnancy as well as pregnant women without diabetes showed a clinically uncomplicated course of pregnancy. In contrast, clinical complications were observed during delivery in 10 out of 29 women with Type 1 diabetes and a restrictive diastolic filling pattern. Due to serious ventricular arrhythmia (ventricular salves $(n=4)$; ventricular tachycardia $(n=3)$ and pathological cardiotocographies of the child, an emergency section had to be carried out in seven women. Labour had to be induced early in three women with progredient dyspnea symptoms of an incipient cardiac decompensation in connection with serious diastolic left ventricular dysfunction. These 10 pregnant women with Type 1 diabetes with clinical complications did not differ from the other pregnant diabetic patients as regards blood pressure or heart rate, nor was proteinuria or edema development documented. Altogether, there were dangerous complications with $34 \%$ (10 out of 29) women.

\section{Discussion}

Pregnancy causes characteristic usually reversible changes in a woman's cardiovascular system due to an increase in total body water by 40 to $100 \%$ and blood volume by 30 to $50 \%$ [16].

Left ventricular function could be assessed by recording the velocity of flow through the mitral valve and pulmonary veins. The mitral inflow pattern is affected by a complex interaction of many factors, including myocardial relaxation, ventricular compliance, preload and afterload, myocardial contractility and pericardial restraint [17]. Pregnancy in healthy women causes an $40 \%$ increase in the preload [4]. This study characterizes the left ventricular response 
to the chronic volume overload state in healthy and diabetic pregnant women. Our data confirm previous observations $[8,18]$ in healthy pregnant women with an increase in the heart rate, cardiac output, cardiac index and left ventricular mass index, as well as a decrease in systemic vascular resistance during pregnancy. Left ventricular ejection fraction and fractional shortening decreased in both groups in the third trimester in accordance with earlier studies [7, 19]. Other studies, however, described an unchanged left ventricular ejection fraction during the entire course of pregnancy $[5,20]$. The left ventricular enddiastolic and endsystolic dimensions did not change during or after pregnancy. This result of our study agrees [6] and differs from those of other investigators $[1,5]$. The slight increase in atrial size during pregnancy has already been documented [1] and it was interpretated as an indirect indicator of the left ventricular filling status. The increase in atrial diameter during pregnancy, as documented by ourselves and others [5, 7], suggests that an increase occurs in both the preload and the circulating blood volume.

The increase of left ventricular wall thickness and left ventricular mass developed in both groups in the second trimester with a maximum in the third trimester. Cardiac hypertrophy occurs in a variety of conditions and serves to normalize increased values of systolic wall stress. The cardiac mass increase is produced by preload augmentation and a successive increase in stroke volume (volume-overload). This theory is supported by the decrease in left ventricular mass 8 weeks after delivery. The cardiovascular changes during pregnancy represent one of the forms of physiological hypertrophy and resemble those found with exercise training in long-distance runners [21].

Despite the increase of the left ventricular mass healthy pregnant women showed a normal left ventricular filling pattern just before delivery [8]. In contrast, in pregnant diabetic women changes of the diastolic filling pattern were documented in the sense of a decrease of the mitral flow in the early filling phase and a compensatory increase of the left ventricular inflow in the late diastolic filling period. This impairment of early diastolic filling velocity normally shows up as an early sign of a cardiac dysfunction even before there is a systolic disturbance along with a loss of contractility [8]. Several investigators have shown that abnormalities of left ventricular diastolic function are common even in diabetic patients without clinical manifestations of congestive heart failure [22, 23]. Whether these abnormalities result from a microangiopathic process in the heart or from metabolic abnormalities inherent to diabetes mellitus is still unclear [24]. Furthermore, it has been suggested that diastolic abnormalities could be an early manifestation of a specific diabetic heart disease [22]. Diastolic dysfunction in diabetic patients has been reported by numerous authors; prevalence of diastolic abnormalities varies from 21 to $100 \%$ in other studies [25, 26]. This variability is probably due to different selection criteria and methods for evaluating diastolic function. Our results show a disturbed relaxation pattern in all investigated diabetic patients already at the first examination. These results of our study are in accordance with those of other authors [22], who found a decreased peak diastolic rate of dimensional change (peak negative $\mathrm{dD} / \mathrm{dt}$ ) in patients with diabetes mellitus they observed a correlation between the degree of diastolic abnormalities and the severity of microvascular complications in patients with diabetes mellitus. These observations and the results of our study indicate that, even when systolic function is not impaired, diabetic patients can show abnormal left ventricular diastolic function potentially related to diabetic microangiopathy $[27,28]$. Diabetic patients had a higher atrial contribution to left ventricular filling and prolonged isovolumetric relaxation and deceleration time than control subjects.

Serious difficulties arise from the fact that the transmitral flow velocity pattern is modified by different preload and afterload conditions. An increase in the ratio of early to late peak velocity with increasing left atrial pressure could have been shown before [15]. In order to exclude that a normalization of the transmitral flow velocity pattern could occur under the condition of an increased filling pressure, we investigated pulmonary vein velocities and "pseudonormalization" could be excluded in all diabetic patients and the control subjects.

In $57 \%$ of the pregnant diabetic patients, a deterioration of diastolic dysfunction was documented at the end of the second trimester, they developed a restrictive filling pattern even before labour and delivery. The coronary reserve decreases and the pressure volume loop is shifted to a less favourable work-level in diabetic women with pre-existing relaxation delay and reduced left ventricular compliance due to deterioration of the passive diastolic qualities of the left ventricle. These pathophysiological mechanisms are described as a risk constellation for potentially dangerous arrhythmia [29]. During delivery, which is marked by an additional pressure and volume overload dangerous complications were documented in one third of these pregnant women with Type 1 diabetes. In former clinical studies, it could be proven that a restrictive diastolic filling pattern is strained in patients with coronary cardiac disease and dilative cardiomyopathy with a bad clinical prognosis due to cardiovascular death and ventricular arrhythmia $[15,30]$. In the course of delivery (marked by pressure and volume load), we documented dangerous complications in $34 \%$ of the pregnant diabetic women who had already developed an early restrictive filling pattern. The early development of a restrictive filling pattern appears to be predictive for dangerous arrhythmia and cardiovascular problems in the course of pregnancy and delivery. 
Only by means of emergency incision and early inducement of delivery, these clinical complications could be mastered.

It can, therefore, be summarised that already an uncomplicated pregnancy in healthy women with normal left ventricular systolic function as a chronic volume overload state has important effects on haemodynamic functional parameters in each trimester. The cardiac dysfunction described is a transient rather than a permanent phenomenon. The volume-overload in a normal pregnancy leads to reversible physiological left ventricular hypertrophy, and to a significant change in left ventricular diastolic function in the sense of a disturbed relaxation pattern. In one third of pregnant women with insulin-dependent diabetes mellitus, changes were documented in the sense of a restrictive filling pattern already during the 2nd trimester. The assessment of left ventricular diastolic function in pregnant women may predict complicated pregnancies. It should be considered to evaluate left ventricular diastolic parameters routinely during pregnancies in women with insulin-dependent diabetes mellitus. Further investigations have to be made to decide whether a natural delivery in these women should be avoided and maybe an elective Caesarean section should be preferred in order to avoid the additional pressure-overload during delivery.

\section{References}

1. Duvekot JJ, Peeters LH (1994) Maternal cardiovascular hemodynamic adaptation to pregnancy. Obstet Gynecol Surv 49 [12 Suppl]:S1-S14

2. Brunazzi MC, Chirillo F, Pasqualini M, Gemelli M et al. (1994) Estimation of left ventricular diastolic pressures from precordial pulsed-Doppler analysis of pulmonary venous and mitral flow. Am Heart J 128:293-300

3. Cecconi M, Manfrin M, Zanoli R, Colonna P, Ruga O, Pangrazi A, Soro A (1996) Doppler echocardiographic evaluation of left ventricular end-diastolic pressures in patients with coronary artery disease. J Am Soc Echocardiogr 9:241-250

4. Hunter S, Robson SC (1992) Adaptation of the maternal heart in pregnancy. Br Heart J 68:540-543

5. Katz R, Karliner JS, Resnik R (1978) Effects of a natural volume overload state (pregnancy) on left ventricular performance in normal human subjects. Circulation 58:434 441

6. Mabie WC, DiSessa TG, Crocker LG, Sibai BM, Arheart KL (1994) A longitudinal study of cardiac output during human pregnancy. Am J Obstet Gynecol 170:849856

7. Robson SC, Hunter S, Boys RJ, Dunlop W (1989) Serial study of factors influencing changes in cardiac output during human pregnancy. Am J Physiol 256:H1060H1065

8. Schannwell CM, Schoebel FC, Zimmermann $\mathrm{T}$ et al. (2000) Linksventrikuläre diastolische Funktion in der normalen Schwangerschaft. Dtsch Med Wochenschr 123:957964
9. Schannwell CM, Heggen S, Schoebel FC et al. (1999) Frühzeitige Einschränkung der diastolischen Funktion bei jungen Typ-I-Diabetikern als Erstmanifestation einer diabetischen Herzmuskelerkrankung. Z Kardiol 88:338-346

10. Sahn DJ, De Maria A, Kisslo J, Weyman A (1978) The committee on M-mode standardization of the American Society of Echocardiography. Recommendations regarding quantification in M-mode echocardiography: results of a survey of echocardiographic measurements. Circulation 58:1072-1081

11. Devereux RB, Casale PN, Eisenberg RR, Miller DH, Kligfield P (1984) Electrocardiographic detection of left ventricular hypertrophy using echocardiographic determination of left ventricular mass as the reference standard. J Am Coll Cardiol 3:82-87

12. Quinones MA, Waggoner AD, Reduto LA et al. (1981) A new, simplified and accurate method for determining ejection fraction with two-dimensional echocardiography. Circulation 64:744-753

13. Oh JK, Appleton CP, Hatle LK, Nishimura RA, Seward JB, Tajik AJ (1997) The noninvasive assessment of ventricular diastolic function with two-dimensional and Doppler echocardiography. J Am Soc Echocardiogr 10:246-270

14. Gentile F, Mantero A, Lippolis A et al. (1997) Pulmonary venous flow velocity patterns in 143 normal subjects aged 20 to 80 years old. Eur Heart J 18:148-164

15. Appleton CP (1997) Hemodynamic determinants of Doppler pulmonary venous flow velocity components: new insights from studies in lightly sedated normal dogs. J Am Coll Cardiol 30:1562-1574

16. McAnulty JH, Metcalfe J, Ueland K (1990) Heart disease and pregnancy. In: Hurst JW, Schlant RC, Rackley CE, Sonnenblick EH, Wenger NK (eds) The heart, arteries and veins, 7th edn. McGraw-Hill, New York, pp 14651478

17. Nishimura RA, Abel MD, Hatle LK et al. (1989) Significance of Doppler indices of diastolic filling of the left ventricle: comparison with invasive hemodynamics in a canine model. Am Heart J 118:1248-1258

18. Mesa A, Jessurun C, Hernandez A et al. (1999) Left ventricular diastolic function in normal human pregnancy. Circulation 99:511-517

19. Laird-Meeter K, van de Ley G, Bom TH, Wladimiroff JW, Roelandt J (1979) Cardiocirculatory adjustments during pregnancy: an echocardiographic study. Clin Cardiol 2:328-332

20. Clapp JF 3rd, Capeless E (1997) Cardiovascular function before, during, and after the first and subsequent pregnancies. Am J Cardiol 80:1469-1473

21. Schannwell CM, Marx R, Plehn G, Perings CH, Leschke M, Strauer BE (2001) Kann eine pathologische linksventrikuläre Hypertrophie bei arterieller Hypertonie von einer physiologischen linksventrikulären Hypertrophie bei Sportlern unterschieden werden? Dtsch Med Wochenschr 126:263-267

22. Shapiro LM, Howat AP, Calter MM (1981) Left ventricular function in diabetes mellitus. Br Heart J 45:122-132

23. Regan TJ, Lyons MM, Ahmed SS et al. (1977) Haider B: evidence for cardiomyopathy in familial diabetes mellitus. J Clin Invest 60:885-899

24. Fein FS, Sonnenblick EH (1994) Diabetic cardiomyopathy. Cardiovasc Drugs Ther 8:65-73

25. Airaksinen KEJ, Koistinen MJ, Ikäheimo MJ (1989) Augmentation of atrial contribution to left ventricular filling in IDDM subjects as assessed by pulsed Doppler echocardiography. Diabetes Care 12:159-161 
26. Airaksinen J, Ikäheimo M, Kaila J, Linnaluoto M, Takkunen J (1984) Impaired left ventricular filling in young female diabetics. An echocardiographic study. Acta Med Scand 216:509-516

27. Strauer BE, Motz W, Vogt M, Schwartzkopff B (1997) Impaired coronary flow reserve in NIDDM. Diabetes 46 [Suppl 2]:8119

28. Strauer BE, Motz W, Vogt M, Schwartzkopff B (1997) Evidence for reduced coronary flow reserve in patients with insulin-dependent diabetes. A possible cause for diabetic heart disease in man. Exp Clin Endocrinol Diabetes 105:15-20
29. Melina D, Colivicchi F, Guerrera G et al. (1992) Prevalence of left ventricular hypertrophy and cardiac arrhythmias in borderline hypertension. Am J Hypertens 5:570-573

30. Pinamonti B, Di Lenarda A, Sinagra G, Camerini F (1993) The Heart Muscle Disease Study Group: restrictive left ventricular filling pattern in dilated cardiomyopathy assessed by Doppler echocardiography: clinical, echocardiographic and hemodynamic correlations and prognostic implications. J Am Coll Cardiol 22:808-815 